\title{
A symplectic method in two dimensional steady state heat conduction problems
}

\author{
Weixiang Zhang ${ }^{1, ~ a, ~ L i ~ C h e n ~}{ }^{2, b}$ \\ ${ }^{1}$ Nantong polytechnic college, Nantong 226002 , PR China \\ ${ }^{2}$ School of Civil Engineering and Architecture, Henan University of Technology, \\ Zhengzhou 450052, PR China \\ aWeixiang zh@163.com, ${ }^{\text {b Lichen@163.com }}$
}

\begin{abstract}
Keywords: Hamiltonian method; Heat conduction; temperature
Abstract. A Hamiltonian method is proposed to solve the steady state heat conduction problem. Due to the completeness property of the solutions, any solution vector can be easily described by linear combination of the fundamental solutions. The method can be used to directly solve various boundary condition problems, including mixed conditions. Numerical results show the decay character of the temperature. The temperature concentration is seriously confined near the boundary of the fixed, and decrease rapidly with the distance of the boundaries.
\end{abstract}

\section{Introduction}

With the fast devlopment of modern science and technology, many researches are closely related with heat conductions or thermo-effects, and thus heat conduction problems gained more and more attention. In recent years, researchers on this subject proposed various theories and computational methods. Zubair obtained an analytic heat conduction temperature and heat flux soluton of a semi-infinite body [1]. Rahimi and Mehryar investigated the effects of duct wall thermal conductivity and thickness on the local nusselt number at the entrance and ending regions of a circular cross-section microchannel in a conjugate heat transfer problem numerically, and the simultaneously developing laminar flow with a constant heat flow rate per unit length on the outside surface of the duct wall is considered [2]. Hetmaniok et al. deal with an application of the homotopy perturbation method for the solution of inverse heat conduction problem. This problem consists in the calculation of temperature distribution in the domain, as well as in the reconstruction of functions describing the temperature and heat flux on the boundary, when the temperature measurements in the domain are known [3]. Ochiai presented the development of the triple reciprocity method for solution of axial symmetric stationary heat conduction problems in continuously non-homogeneous media with eliminating the domain integrals. In this method, the spatial variations of domain "sources" are approximated by introducing new potential fields and using higher order fundamental solutions of the Laplace operator [4]. In the work of Ma et al. [5], an anisotropic heat transfer numerical model is proposed to analyze the local thermal field of primary surface plate affected by the small-scale longitudinal heat conduction. Moreover, a detached numerical model is used for simplifying grid and improving computational stability. It shows that the temperature distribution is non-uniform in the anisotropic heat transfer model. Feng and Gao [6] presented a new single interface integral equation method for solving transient heat conduction problems consisting of multi-medium materials with variable thermal properties. Firstly, adopting the fundamental solution for the Laplace equation, the boundary-domain integral equation for transient heat conduction in single medium is established. Then from the established integral equation, a new single interface integral equation is derived for transient heat conduction in general multi-medium functionally graded materials, by making use of the variation feature of the material properties. Zhai et al. developed a theoretical model is to investigate the effects of the axial heat conduction on the laminar forced convection in a circular tube with uniform internal heat generation in the solid wall [7]. 
However the above mentioned methods are based on the temperature as the fundamental function, belongs to a Lagrange system. It is difficult to give the heat flux solution directly. Zhong [8] introduced the Hamiltonian formulation into the theory of elasticity and put forward a direct method. In this paper, the Hamiltonian system is introduced into steady state heat conduction problem. In the prensent approach, by the temperature and heat flux functions are selected to be funamental variables, the governing equation of the Hamiltonian system is established, and hence the problem is transformed to finding eigenvalues and cooresponding eigensolutions.

\section{The steady-state heat conduction problems}

In the rectangular coordinate system, consider a strip plane whose length and width is $2 l$ and $2 b$, respectively. According to the Fourier law, the heat flux density can be expressed as

$\varphi_{z}(z, x)=-k \partial_{z} T, \quad \varphi_{x}(z, x)=-k \partial_{x} T$

where $k$ is the coefficient of heat conduction, $T$ is the temperature funtion, and $\varphi$ is the density of heat flux. The Lagrangian function is

$$
L(T, \hat{\mathcal{K}})=-\left[\left(\partial_{x} T\right)^{2}+\mathcal{K}^{2}\right] / 2
$$

where an over dot on a variable represents its partial differential to coordinate $z$. Based on the Hamiltonian principle, we have

$$
\delta \int_{-l}^{l} \int_{-b}^{b} L(T) d x d z=0
$$

The dual variable of the temperature funtion is just the density of heat flux along coordinate $z$

$$
\varphi=\varphi_{z}=\frac{\partial L}{\partial \mathcal{R}^{\alpha}}=-k \mathcal{\alpha}
$$

According to the variation principle, we can derive the governing equations in the Hamiltonian system

$$
\hat{\alpha}=\frac{\partial H}{\partial \phi}=-\frac{1}{k} \varphi, \quad \Leftrightarrow \ll=-\frac{\partial H}{\partial T}=k \partial_{x}^{2} T
$$

\section{Fundamental eigensolutons}

The general solution can be obtained by solving the equation (5) directly

$$
\psi_{j}=\left\{\begin{array}{c}
1 \\
-k \mu
\end{array}\right\}\left[C_{1} \cos (\mu x)+C_{2} \sin (\mu x)\right]
$$

where $\mu$ is an eigenvalule, and $\psi$ is the cooresponding eigensoluton. Substituding equation (6) to heat insulation condition, one has 


$$
\begin{aligned}
& -C_{1} \sin (\mu b)+C_{2} \cos (\mu b)=0 \\
& -C_{1} \sin (-\mu b)+C_{2} \cos (-\mu b)=0
\end{aligned}
$$

Non-trivial solution of Eq. (7) exist if and only if the determinant of the coefficient matrix is zero, and by solving the equation, we get a series of eigenvalues. For the cases of positive eigenvalues and the negative ones, the solution can be expressed as

$$
\left.\psi_{n}^{\alpha}=\left[\begin{array}{c}
1 \\
-\frac{k n \pi}{2 b}
\end{array}\right] \cos \frac{n \pi x}{2 b}\right)+\left[\begin{array}{c}
1 \\
-\frac{k n \pi}{2 b}
\end{array}\right] \sin \frac{n \pi x}{2 b}
$$

and

$$
\psi_{n}^{\beta}=\left[\begin{array}{c}
\frac{1}{k n \pi} \\
\frac{1}{2 b}
\end{array}\right] \cos \frac{n \pi x}{2 b}+\left[\begin{array}{c}
\frac{1}{k n \pi} \\
\frac{1}{2 b}
\end{array}\right] \sin \frac{n \pi x}{2 b}
$$

respectively. Now, let's discuss the conditions that left end supplies stresses while right end offers displacements, that is

$$
\begin{aligned}
& \left.T\right|_{z=l}=\left.T_{l}(x) \quad \varphi\right|_{z=l}=\varphi_{l}(x) \\
& \left.T\right|_{z=-l}=\left.T_{-l}(x) \quad \varphi\right|_{z=-l}=\varphi_{-l}(x)
\end{aligned}
$$

Applying the boundary integral law and the fundamental eigensolutions, equations about the eigensolution expansion are obtained as

$$
\begin{aligned}
& \sum_{n=0} a_{n} T_{n}^{(\alpha)}(x) e^{\mu_{n} z}+\sum_{n=0} b_{n} T_{n}^{(\beta)}(x) e^{-\mu_{n} z}=T(x, z) \\
& \sum_{n=0} a_{n} \varphi_{n}^{(\alpha)}(x) e^{\mu_{n} z}+\sum_{n=0} b_{n} \varphi_{n}^{(\beta)}(x) e^{-\mu_{n} z}=\varphi(x, z)
\end{aligned}
$$

So the combination of non-zero eigenvalue solution is uniquelydetermined. 


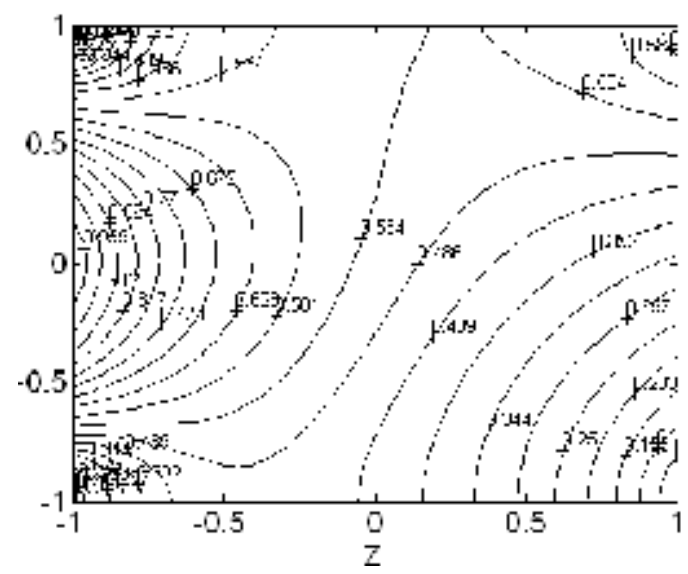

Fig.1 The contours of temperature

\section{Numerical results}

As an example to show the stress concentrations, we discuss the following boundary conditions problem:

$$
T_{-l}=1-x^{2}, T_{l}=(x+1) / 3
$$

Figure 1 shows the distribution of temperature. The figure explains that the local effects of the end boundary appear due to the restraints of boundary conditions. From the figure, we can see that in the reigion far from the boundary, the temperature keeps nearly a constant, while near the boundary, the teperature appears nonuniform property. The results well explained the Saint-Venant principle.

\section{Conclusions}

With the direct method, all the Saint-Venant solutions have been derived from the zero eigenvalue solutions. In equivalent force systems, the solutions can satisfy effective boundary conditions only and describe characters of the creep and relaxation wholly in viscoelasticity. Nevertheless, these solutions are obvious incomplete in the whole solution space. The Hamiltonian equation must have others non-zero eigenvalue solutions. The eigensolutions can express local effect near boundarys. In the discussion of local effect, only the first few eigensolutions play important role. In fact, the solution of problem should be composed of zero eigenvalue solutions and non-zero eigenvalue solutions, which is close form.

\section{References}

[1] S.M. Zubair and M.A.Chaudhry: Int.J. Heat Mass Tran Vol. 38 (1995), p. 3393

[2] M. Rahimi and R. Mehryar: Int. J. Therm. Sci Vol. 59 (2012), p. 87

[3] E. Hetmaniok, I. Nowak, D. Słota D, and R. Wituła: Int. Commun. Heat Mass Vol. 39 (2012), p. 30

[4] Y. Ochiai, V. Sladek, and J. Sladek: Eng. Anal. Bound. Elem Vol. 37 (2013), p. 336

[5] T. Ma, J. Zhang, S. Borjigin, Y.T. Chen, Q.W. Wang, and M.Zeng: Appl. Therm.Eng. Vol. 76 (2015), p. 272

[6] W.Z. Feng and X.W. Gao: Int. J. Heat Mass Tran Appl. Therm. Vol. 98 (2016), p. 227 
[7] L.J. Zhai, G.Q. Xu, Y.K. Quan, G. Song, B. Dong, and H.G. Wu: Int. J. Heat Mass Tran Appl. Therm. Vol. 114 (2017), p. 238

[8] W.X. Zhong: Duality system in applied mechanics and optimal control. Kluwer Academic Publications, Norwich, NY (2004) 